\title{
ARTICLE Multiparametric mapping of white matter microstructure in catatonia
}

Jakob Wasserthal ${ }^{1,2}$, Klaus H. Maier-Hein $\mathbb{D}^{1,3}$, Peter F. Neher ${ }^{1,2}$, Georg Northoff ${ }^{4}$, Katharina M. Kubera ${ }^{5}$, Stefan Fritze ${ }^{6}$, Anais Harneit ${ }^{7}$, Lena S. Geiger ${ }^{7}$, Heike Tost (i) $^{7}$, Robert C. Wolf $\mathbb{1 D}^{5}$ and Dusan Hirjak ${ }^{6}$

Catatonia is characterized by motor, affective and behavioral abnormalities. To date, the specific role of white matter (WM) abnormalities in schizophrenia spectrum disorders (SSD) patients with catatonia is largely unknown. In this study, diffusion magnetic resonance imaging (dMRI) data were collected from 111 right-handed SSD patients and 28 healthy controls. Catatonic symptoms were examined on the Northoff Catatonia Rating Scale (NCRS). We used whole-brain tract-based spatial statistics (TBSS), tractometry (along tract statistics using TractSeg) and graph analytics (clustering coefficient-CCO, local betweenness centralityBC) to provide a framework of specific WM microstructural abnormalities underlying catatonia in SSD. Following a categorical approach, post hoc analyses showed differences in fractional anisotrophy (FA) measured via tractometry in the corpus callosum, corticospinal tract and thalamo-premotor tract as well as increased CCO as derived by graph analytics of the right superior parietal cortex (SPC) and left caudate nucleus in catatonic patients (NCRS total score $\geq 3 ; n=30$ ) when compared to non-catatonic patients (NCRS total score $=0 ; n=29$ ). In catatonic patients according to DSM-IV-TR $(n=43)$, catatonic symptoms were associated with FA variations (tractometry) of the left corticospinal tract and CCO of the left orbitofrontal cortex, primary motor cortex, supplementary motor area and putamen. This study supports the notion that structural reorganization of WM bundles connecting orbitofrontal/ parietal, thalamic and striatal regions contribute to catatonia in SSD patients.

Neuropsychopharmacology (2020) 45:1750-1757; https://doi.org/10.1038/s41386-020-0691-2

\section{INTRODUCTION}

Catatonia is a psychomotor syndrome characterized by hypo- and hyperkinetic motor phenomena, affective symptoms, and disorders of behavior [1-8]. Magnetic resonance imaging (MRI) studies showed that catatonia in schizophrenia spectrum disorders (SSD) is associated with aberrant frontoparietal, thalamic and cerebellar networks [9-14]. Since catatonia is very likely to be associated with dysconnectivity of large-scale neural networks [15], dysconnectivity may be traced to abnormal white matter (WM) microstructure. At least two lines of evidence support this claim: First, numerous case studies have showed that WM abnormalities in the context of immune dysregulation can lead to catatonia [16]. Second, altered myelin-specific gene expression and neurochemical aberrations (e.g., low-grade inflammation/ neurodegeneration) are associated with more severe catatonic symptoms $[17,18]$.

Here, we combined three distinct diffusion-tensor imaging (DTI) analysis techniques with an established diagnostic approach $[10,11]$ to specifically investigate WM microstructural alterations which are related to catatonia in SSD. First, we used a whole-brain voxel-wise analysis of fractional anisotropy (FA) in catatonia using Tract-Based Spatial Statistics (TBSS) [19]. TBSS projects all subjects' diffusivity data onto a mean FA tract skeleton, before applying voxel-wise cross-subject statistics. However, coregistration algorithms used in voxel-based analysis have difficulties in accurately aligning fiber tracts due to variation in tracts size and shape $[20,21]$. TBSS tries to reduce this problem by calculating statistics on a "voxel skeleton" but is still affected by coregistration inaccuracies. Therefore, in a second step, we used a recently developed comprehensive approach (TractSeg) [22] which allows an accurate reconstruction of fiber tracts in subject space, thus avoiding the problem of inaccurate coregistration of tracts with varying size and shape. We performed statistical analyses by evaluating FA along each of the 15 neurobiologically plausible tracts (often referred to as Tractometry) which represent important connections between sensorimotor regions [corpus callosum (rostrum (CC 1), genu (CC 2), rostral body (CC 3), anterior midbody (CC 4), posterior midbody (CC 5), isthmus (CC 6), splenium (CC 7)), corticospinal tract (CST), thalamo-premotor (T_PREM), striato-fronto-orbital (ST_FO), and striato-premotor (ST_PREM)]. Third, we analyzed network variations in the structural connectome. We employed the concepts of local network clustering coefficient (CCO) and local network betweenness centrality $(\mathrm{BC})$ to pursue hypothesis-driven and locally specific

\footnotetext{
${ }^{1}$ Division of Medical Imaging Computing (MIC), German Cancer Research Center (DKFZ), Heidelberg, Germany; ${ }^{2}$ Medical Faculty Heidelberg, Heidelberg University, Heidelberg,

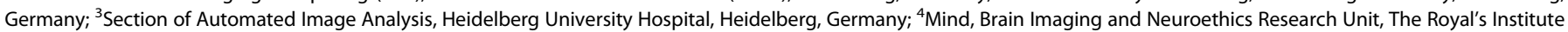

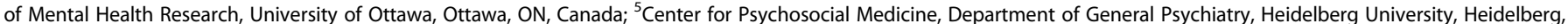

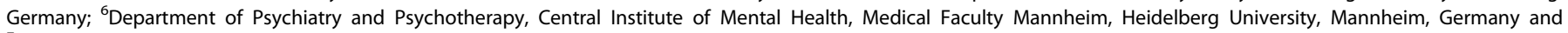

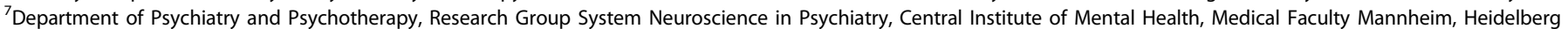
University, Mannheim, Germany

Correspondence: Dusan Hirjak (dusan.hirjak@zi-mannheim.de)
}

Received: 1 March 2020 Revised: 20 April 2020 Accepted: 22 April 2020

Published online: 5 May 2020 
analyses of network indices assessed for psychomotor regions [orbitofrontal cortex (OFC), primary motor area (M1), supplementary motor area (SMA), superior parietal cortex (SPC), thalamus, nucleus caudatus and putamen] identified by previous MRI studies [9-14] and case reports $[23,24]$ on catatonia in SSD. While CCO is defined as a ratio of total number of edges among the neighbors of the node (region) to the total number of edges that can exist among the neighbors of the node per node [25], BC of a node represents the number of shortest paths from all vertices to all others that pass through that node [26].

This study had two main objectives: First, conducting a categorical approach [i.e., comparing SSD patients with and without catatonia according to Northoff Catatonia Rating Scale (NCRS) [27]], we predicted that there will be a difference in TBSSspecific FA, the above-mentioned 15 tracts connecting sensorimotor regions, and graph-based large-scale network measures of psychomotor regions (CCO and BC of OFC, M1, SMA, SPC, thalamus, nucleus caudatus, and putamen) between patients with and without catatonia and healthy controls (HC). Second, following a dimensional perspective on catatonia [28, 29], we supposed that distinct dimensions of catatonia-i.e., motor, behavioral and affective domains - will be significantly associated with TBSS-specific FA, the above-mentioned 15 tracts and graphbased large-scale network measures of psychomotor regions.

\section{METHODS}

Patients and healthy controls

We examined a total of 111 right-handed [30] patients satisfying DSM-IV-TR [31] criteria for schizophrenia $(n=104)$ or schizoaffective disorder $(n=7)[10,11]$ and $28 \mathrm{HC}$. Inclusion and exclusion criteria are listed in the supplement. The local Ethics Committee (Medical Faculty at Heidelberg University, Germany) approved the study. Written informed consent was obtained from all SSD patients and $\mathrm{HC}$ after all aims and procedures of the study had been fully explained.

\section{Clinical assessment}

All patients were recruited and examined during inpatient treatment within one week after partial remission of psychotic symptoms. The duration between psychometric testing, motor assessment and MRI examination was less than 3 days. At the time of the psychometric testing, motor assessment and MRI examination, none of the SSD patients had taken benzodiazepines or anticholinergic agents and all but five $(5 / 111=4.5 \%$ antipsychotic-free) patients were on stable antipsychotic medication for at least two weeks. Daily doses of antipsychotic medication were converted to olanzapine equivalents (OLZ) [32]. For the assessment of catatonic symptoms we used the German version of the NCRS [27]. The scale measures the presence and severity of motor (13 items), affective (12 items), and behavioral (15 items) catatonic symptoms.

In a first step, we followed a categorical approach, using the NCRS criteria [27] to cover all three categories of catatonia and to identify a clear cut-off to distinguish subjects with (NCRS total score $\geq 3$; at least 1 point in the three different symptom categories; i.e., motor, behavioral, and affective) and without $(\mathrm{NCRS}=0)$ catatonia (presence vs. absence) [33]. Thirty out of 111 SSD patients $(27 \%)$ were defined as having catatonia (NCRS total score $\geq 3$ ). The patient control group consisted of twenty-nine right-handed SSD patients defined as not having catatonia (NCRS total score $=0$ ) matched for age, gender, education and OLZ equivalents. This approach allowed us to investigate neural underpinnings of catatonia controlling for the effects of SSD $[33,34]$. The HC group consisted of 28 right-handed participants matched for age and gender. In a next step, we followed a dimensional approach and identified forty-three out of 111 SSD patients $(38,7 \%)$, which were defined as having catatonia according to DSM-IV-TR [1 motor and at least 1 other symptom (behavioral or affective)] [31].

While the diagnosis of catatonia according to NCRS is rather conservative in comparison to other criteria, the dimensional approach using the DSM-IV-TR allowed us to identify a larger group of catatonic patients. In this group, we used the NCRS subscale scores to determine the neurobiological continuum in SSD patients with a broader spectrum of the catatonic syndrome (low and high severity) truly reflecting the dimensional view on catatonia [31].

MRI acquisition

MRI scans were acquired at CIMH on a 3.0 Tesla Magnetom TIM Trio MR scanner (Siemens) equipped with a 32 channel multi-array head-coil. Technical details on MRI sequences are provided as Supplementary Information.

Image processing

Preprocessing. T1 images were skull stripped and bias field corrected using FSL $[35,36]$. DWI images were denoised (MRtrix dwidenoise) [37], corrected for Gibbs ringing artifacts (MRtrix mrdegibbs) [38], corrected for eddy currents and head motion (FSL eddy) [39], corrected for bias field (MRtrix dwibiascorrect) [40] and brain masked (FSL bet) [41]. DWI images were rigidly registered to MNI space using FSL FLIRT [42]. T1 images were rigidly registered to the DWI images. Finally, all images were manually inspected and only images were kept where this preprocessing pipeline was successful.

Data analysis. TBSS: To detect global patterns of WM variations, we followed the recent recommendation regarding TBSS [43] and aligned all subjects to a common space using tensor-based registration with DTI-TK (http://www.nitrc.org/projects/dtitk). We used TBSS [19] to build a common WM skeleton from FA images derived from the co-registered tensor images. We then mapped all dMRI measures onto the skeleton using the standard FSL TBSS commands. The design matrix of the general linear model was set up to identify differences in the voxel-wise TBSS-specific FA between SSD patients with and without catatonia and HC. As recommended, we used the "randomise" command for Monte Carlo permutation tests with $n=5000$ repetitions and a confidence threshold of $p<0.05$ for the corrected threshold-free cluster enhancement (TFCE) significance maps [44]. Additionally, for each of the bundles between sensorimotor regions (CC 1-7, CST, T_PREM, ST_FO, and ST_PREM) which were also used for the Tractometry analysis we averaged the FA values of the TBSS WM skeleton within the bundle mask (as generated with TractSeg). These average FA values were used for additional between-group analyses along the lines of Kelly and colleagues [45].

Tractometry: Along-tract FA statistics (Tractometry) were obtained using TractSeg [22, 46]. TractSeg generates bundlespecific tractograms and then evaluates the FA along 100 points of each tract (more details are provided as Supplementary Information). Statistics such as correlations and T-tests can be calculated point-wise along these 100 points. We used the permutation-based multiple comparison correction (with $n=$ 5000 repetitions) published by Nichols and Holmes $(2001)[47,48]$ to appropriately adjust $p$ values given the correlation structure of the data. We used an uncorrected confidence threshold of $p<0.05$ (the corrected $p$ value is different for each bundle depending on its correlation structure). Controlling for covariates was done by regressing them out of the data before calculating the t-test (categorical approach) or correlation (dimensional approach).

Large-scale network analysis: Whole-brain tractography was performed following the recommendations given by the evaluation of fiber tracking algorithms [49] (details are provided as Supplementary Information). While tractography-based approaches typically analyze specific structures of interest, a 


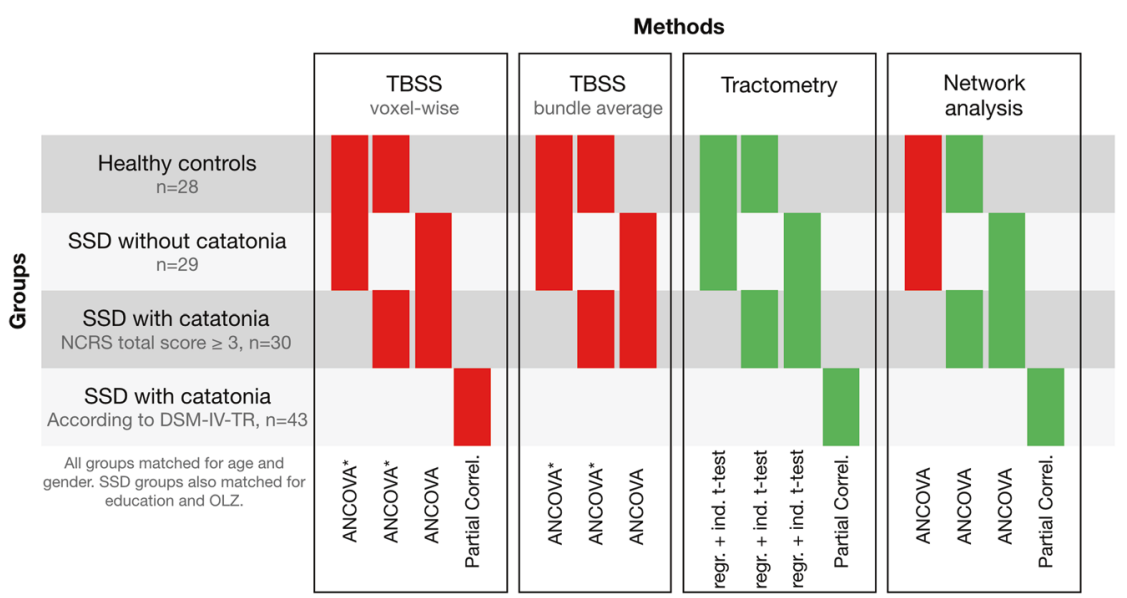

Fig. 1 Overview of the different analytical steps. Colored bars indicated which groups are compared by the respective statistical test. The color shows if the test gave significant results $(p<0.05)$ (green) or not (red). ${ }^{*}$ indicates significant findings from analyses that were not covaried for age, gender, aducation and OLZ. SSD: schizophrenia spectrum disorders, reg. + ind. t-test: regressing out covariates + independent t-test, Partial Correl.: partial correlation.

graph-based large-scale network analysis of the connectome can yield comprehensive measures of larger-scale architectural patterns in the brain $[25,50]$. To construct a graph representation, the T1 image was used to create a parcellation of the brain using FreeSurfer (http://surfer.nmr.mgh.harvard.edu/) [51-53] and the atlas by Destrieux [54-56]. Details on the FreeSurfer processing stream are provided as Supplementary Information. Connectomics networks were consecutively constructed based on both the tractography result and the brain parcellation using MRtrix [57]. We have computed the normalized CC and BC for 7 nodes (OFC, M1, SMA, SPC, thalamus, nucleus caudatus, and putamen) located in both hemispheres. A nominal significance threshold of $p \leq 0.05$ was defined, and $p$ values of the identified associations were corrected for the number of tested nodes in our main analysis using the Bonferroni method. The corrected threshold was set to $p=0.0006[a=0.05 / 80$ tests $(5$ nodes $\times 2$ parameters $\times 2$ hemispheres $\times 4$ NCRS scores)]. For completeness, uncorrected findings at $p<0.05$, will be also reported.

\section{Statistical analyses}

The following statistical tests, including the covariates, were used for all the above-mentioned data analyses [TBSS (voxel-wise and aggregated FA), Tractometry and Large-scale network analysis] (for overview see Fig. 1): Following the categorical approach, we controlled for PANSS total score and BARS global score, because the two study groups differed significantly in these two variables. Duration of illness (DOI), Packyears (PY; number of cigarettes as a potential confounding factor), SAS and AIMS total scores were not included as covariates since (1) these variables did not differ significantly (all $p$ values $>0.05$ ) between the two groups and (2) there was no significant correlation between NCRS total score and SAS total score $(r=0.318 ; p=0.087)$, AIMS total score $(r=$ $0.314 ; p=0.091)$, DOI $(r=-0.037 ; p=0.846)$ and PY $(r=-0.185$; $p=0.329)$ in SSD patients with catatonia $(n=29)$. Still, we performed supplementary between-group analyses using PANSS total score, BARS global score, SAS total score, AIMS total score, DOI, and PY as covariates (see Supplementary Table 3 and Supplementary Fig. 4 for results). In the next step, we also included an additional between-group ANCOVAs [controlling for (i) age, gender, education and OLZ and (ii) age and gender] comparing catatonic and non-catatonic SSD patients with 28 right-handed $\mathrm{HC}$ matched for age $[$ mean $=35.14$ years; $\mathrm{SD}=$ 14.18), $\mathrm{F}_{(2,84)}=0.93, p=0.39$ ] and gender (20 males, 8 females) (Table 1). For the dimensional approach in catatonic patients (two-tailed partial correlation) we controlled for age, gender, medication and PANSS total score.

The selection of different covariates for the categorical and dimensional approaches is based on different hypotheses, the recommendations of the neuroimage community [58, 59], the influence of individual covariates on the target variables (WM metrics and catatonic symptoms) $[60,61]$ and previous MRI studies on motor abnormalities in SSD [10-12, 62]. Finally, we calculated the effect size (Cohen's d) and statistical power (using G*Power) of significant results (for results see Supplementary Material).

\section{RESULTS}

Clinical and demographic characteristics

Clinical and demographic characteristics are shown in Table 1. Thirty SSD patients with and twenty-nine SSD patients without catatonia according to NCRS as well as $28 \mathrm{HC}$ were included in the final between-group (categorical) analyses. Forty-three SSD patients fulfilling the DSM-IV-TR [31] criteria for catatonia were included in the final correlational (dimensional) analyses. In the non-catatonic SSD group, none of the twenty-nine patients would qualify for any of the single DSM-IV-TR criteria of catatonia. Thirtynine SSD patients $(39 / 111=35.1 \%)$ who did not meet the NCRS or DSM-IV-TR criteria for catatonia or could not have been included in the matched control group were excluded from further analyses.

\section{Categorical analyses}

TBSS. The standard TBSS analyses did reveal a nominal reduction of FA in catatonic patients compared to noncatatonic patients, but this difference was not statistically significant $(p=0.19)$. Using the average FA across the 15 tracts between sensorimotor regions, ANCOVA revealed no significant difference between catatonic and non-catatonic SSD patients (all $p$ values $>0.1$ ). After excluding the four covariates there was still no significant difference in FA between catatonic and noncatatonic patients (all $p$ values $>0.08$ ).

Tractometry. Looking at along-tract statistics (Tractometry) using TractSeg, we found significantly decreased FA in the corpus callosum (CC) (min. $p$ values $<0.002$ ) and significantly increased FA in the thalamo-premotor tract (T_PREM) $(\min . p$ value $=0.0006)$ (all $p$ values below alphaFWE corrected threshold) in catatonic 
Table 1. Demographic and clinical variables in SSD patients with and without catatonia according to NCRS and healthy controls.

\begin{tabular}{|c|c|c|c|c|c|c|}
\hline & $\begin{array}{l}\text { Patients with } \\
\text { catatonia }(n=30)\end{array}$ & $\begin{array}{l}\text { Patients without } \\
\text { catatonia }(n=29)\end{array}$ & $\begin{array}{l}\text { Healthy controls } \\
(n=28)\end{array}$ & $T^{a} / F^{b}$ & $D f^{a / b}$ & Sig. $(2 \text {-tailed })^{a / b}$ \\
\hline Age & $39.40 \pm 10.49$ & $38.00 \pm 11.25$ & $35.14 \pm 14.18$ & $0.494 \mathrm{~F}(2,84)=0.934$ & 57 & $0.623 / 0.397$ \\
\hline Education (years) & $13.77 \pm 2.41$ & $13.17 \pm 3.12$ & $15.39 \pm 1.4$ & $0.818 F(2,84)=6.343$ & 57 & $0.417 / 0.003$ \\
\hline Olanzapine equivalents & $18.03 \pm 9.64$ & $18.41 \pm 12.51$ & 0 & $-0.133 F(2,84)=37.378$ & 57 & $0.894 /<0.0001$ \\
\hline Packyears & $12.02 \pm 13.06$ & $11.48 \pm 10.23$ & n.a. & 0.175 & 57 & 0.861 \\
\hline PANSS total score & $80.27 \pm 20.73$ & $56.72 \pm 19.42$ & n.a. & 4.497 & 57 & $<0.001$ \\
\hline PANSS positive score & $18.93 \pm 8.18$ & $13.1 \pm 6.16$ & n.a. & 3.083 & 57 & 0.003 \\
\hline PANSS negative score & $21.17 \pm 8.63$ & $13.83 \pm 6.75$ & n.a. & 3.628 & 57 & 0.001 \\
\hline PANSS global score & $40.4 \pm 11.85$ & $29.9 \pm 9.64$ & n.a. & 3.725 & 57 & $<0.001$ \\
\hline NCRS affective score & $2.8 \pm 1.66$ & 0 & n.a. & 9.031 & 57 & $<0.001$ \\
\hline NCRS behavior score & $2.27 \pm 1.2$ & 0 & n.a. & 10.156 & 57 & $<0.001$ \\
\hline NCRS total score & $6.9 \pm 2.6$ & 0 & n.a. & 14.263 & 57 & $<0.001$ \\
\hline SAS total score & $3.27 \pm 2.24$ & $2.14 \pm 2.13$ & n.a. & 1.979 & 57 & 0.053 \\
\hline AIMS total score & $1.83 \pm 3.05$ & $0.59 \pm 1.93$ & n.a. & 1.867 & 57 & 0.067 \\
\hline BARS global score & $1.2 \pm 1.51$ & $0.48 \pm 0.98$ & n.a. & 2.144 & 57 & 0.036 \\
\hline
\end{tabular}

compared to non-catatonic patients (Fig. 2). Regarding the corticospinal tract (CST), we found significant differences (both decrease and increase) in FA values depending on the location of the bundle between catatonic and non-catatonic patients (min. $p$ value $=0.0007$; Fig. 2 and Supplementary Fig. 1).

Large-scale network analysis. We found significantly higher CCO of the left caudate nucleus in catatonic compared to noncatatonic patients $(p=0.03)$ (Supplementary Table 1).

Correlational (dimensional) analyses

TBSS. We found no significant association between NCRS scores and FA (voxel-wise TBSS) (all $p$ values $>0.05$ ).

Tractometry. We found a significant positive association between NCRS behavioral score and FA in the left CST ( $\min . p$ value $=$ 0.0006; all $p$ values below alphaFWE corrected threshold) (Fig. 3).

Large-scale network analysis. We found a significant association between NCRS motor score and CCO of the left OFC $(p=0.006)$, M1 $(p=0.04)$ and putamen $(p=0.01)$ as well as CCO and BC of the right SMA ( $p=0.04$ and $p=0.0003$ ) (Table 2). Further, we found a significant association between NCRS total score and CCO of the left OFC $(p=0.04)$ and BC of the right thalamus $(p=0.01)$ (Table 2). However, only the association between NCRS motor score and $\mathrm{BC}$ of the right SMA survived the Bonferroni correction for multiple testing (threshold at $p=0.0006$ ).
Patients vs. healthy controls

TBSS. The standard voxel-wise TBSS analyses did not reveal significant differences of FA in SSD patients with and without catatonia when compared to $\mathrm{HC}(p=0.74$ and $p=0.59)$. Using the average FA across the 15 tracts between sensorimotor regions, ANCOVA revealed no significant difference between catatonic patients and $\mathrm{HC}$ (all $p$ values $>0.5$ ) and non-catatonic patients and $\mathrm{HC}$ (all $p$ values $>0.2$ ). Using ANCOVA with age and gender as covariates, we found significantly lower FA in CC6 $(p=0.03)$ and CC7 $(p=0.03)$ of catatonic patients when compared to HC. We also found significantly lower FA in CC1 $(p=0.04)$ and right ST_FO $(p=0.04)$ of non-catatonic patients when compared to $\mathrm{HC}$. After excluding all four covariates (age, gender, education and OLZ) and performing an independent two sample t-test, we found significantly lower FA in all CC regions (all $p$ values $<0.05)$ and left $(p=0.03$ ) and right $(p=0.01)$ ST_FO of catatonic patients when compared to HC. We also found significantly lower FA in CC2 ( $p=$ $0.03)$ and ST_PO $(p=0.01)$ of non-catatonic patients when compared to $\mathrm{HC}$.

Tractometry. We found significantly decreased FA in the CC, left and right T_PREM, left and right ST_FO and both ST_PREM in catatonic patients when compared to HC (Supplementary Fig. 2). There was also a significant difference in FA of the CC, left and right CST, right ST_FO and left ST_PREM between non-catatonic patients and HC (Supplementary Fig. 3). 

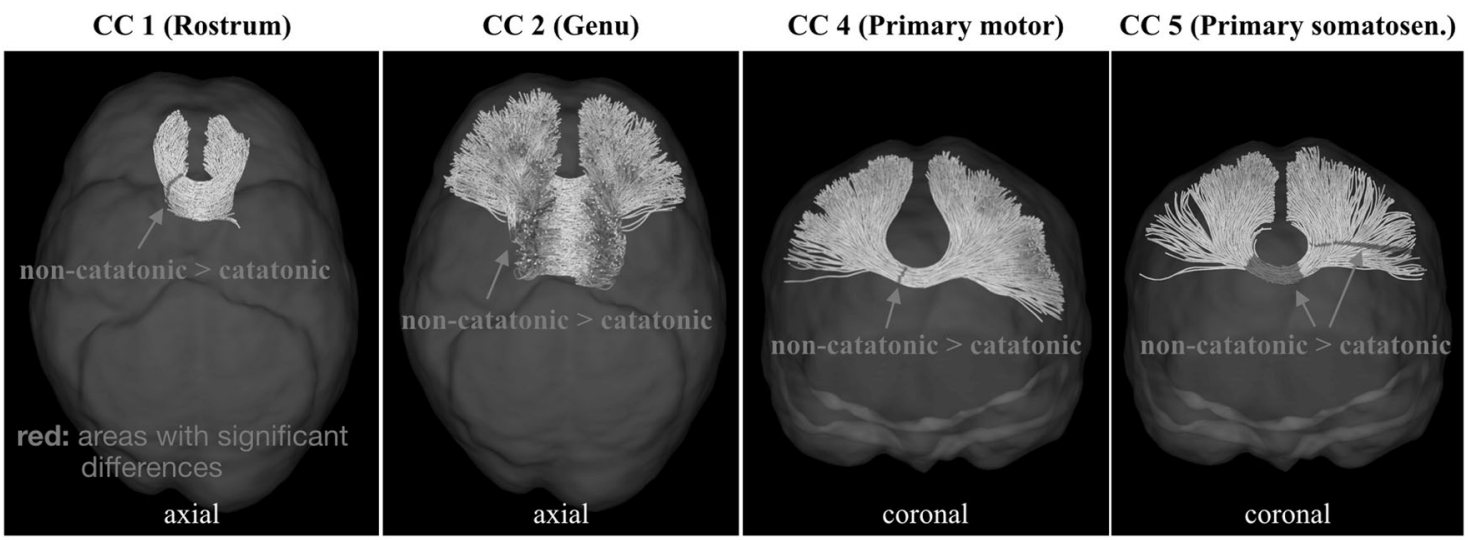

right T_PREM (Thalamo-premotor)

left CST (Corticospinal tract)
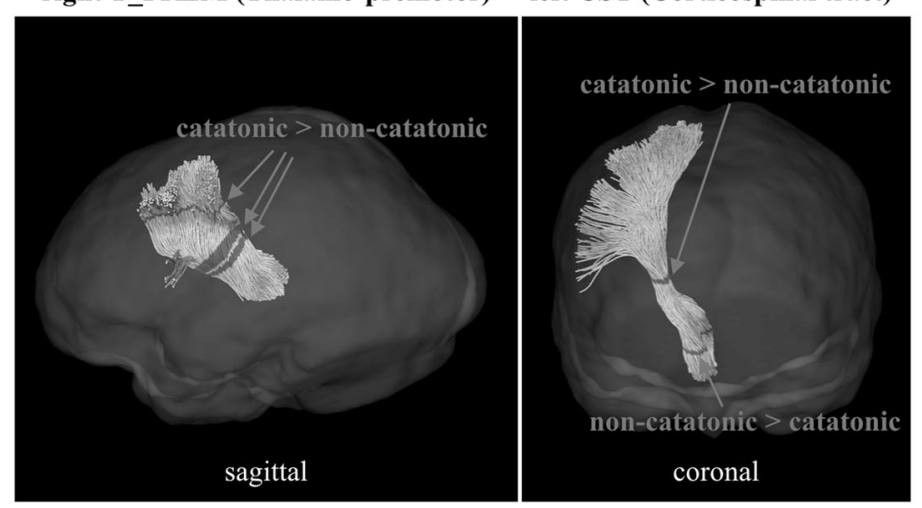

Fig. 2 Significant differences in FA between non-catatonic and catatonic SSD patients (Tractometry analysis).

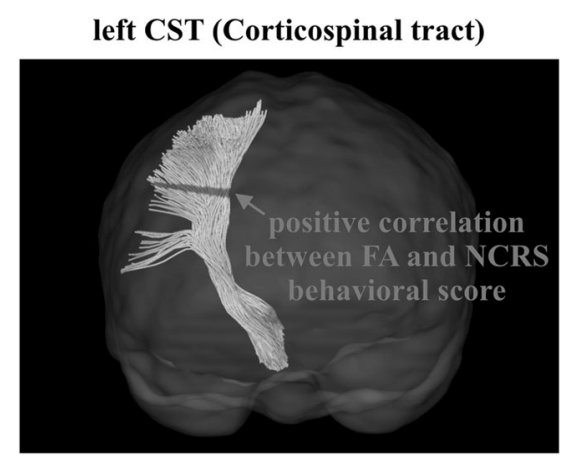

Fig. 3 Significant correlations between FA variations and NCRS behavioral score in catatonic patients (Tractometry analysis).

Large-scale network analysis. We found significantly higher BC of the right supplementary motor area $(p=0.01)$ as well as higher CCO of the right superior parietal cortex and putamen in catatonic patients compared to HC (Supplementary Table 2).

\section{DISCUSSION}

This dMRI study investigated brain WM microstructure abnormalities underlying catatonia in SSD patients. Three main findings emerged: First, following a categorical approach, we found significantly reduced FA in CC and higher FA in T_PREM in catatonic compared to non-catatonic SSD patients. There was a location-dependent increase and decrease of FA in CST in catatonic compared to non-catatonic SSD patients. Second, we found a significant positive association between NCRS behavioral score and FA in the left CST. Third, catatonic symptoms were significantly associated with variations of local network indices in the OFC, M1, SMA, thalamus and putamen.

TBSS and Tractometry

Standard voxel-based TBSS could not identify significant differences in FA between catatonic and non-catatonic patients or significant associations between FA alterations and catatonic symptoms. Since FA alterations are a marker of both myelination and/or axonal disruption [63] and WM tracts organization [64-66], the anomalies that could be considered as catatonia-associated were not severe enough to be detected by voxel-based TBSS. Given that catatonic symptoms are considered to be a "specifier" of SSD according to DSM-5 and appear as transient phenomena, this notion seems plausible.

While we did not find any significant FA differences between SSD patients and HC using the standard voxel-wise TBSS, we used the average $F A$ across the 15 tracts between sensorimotor regions (similar to a large-scale coordinated study of WM microstructural differences in schizophrenia conducted by Kelly and colleagues [45]). Using an ANCOVA, we did not find any significant betweengroup differences. After excluding the four covariates age, gender, education and OLZ from the statistical analyses, we identified significantly lower FA in CC and striato-fronto-orbital tract in catatonic patients when compared to HC. Thus we were able to corroborate the results of Kelly and colleagues [45]. In addition, our findings provide further evidence for the microstructural dysconnectivity in SSD patients with catatonia.

Using Tractometry with TractSeg [22] we identified significantly reduced FA in the CC and CST as well as increased FA in the T_PREM tract and CST in catatonic compared to non-catatonic SSD patients. These results are important for several reasons: First, CC is essential for interhemispheric transfer in terms of increased inhibition or synchronization of bilateral movements [67]. Poggi 


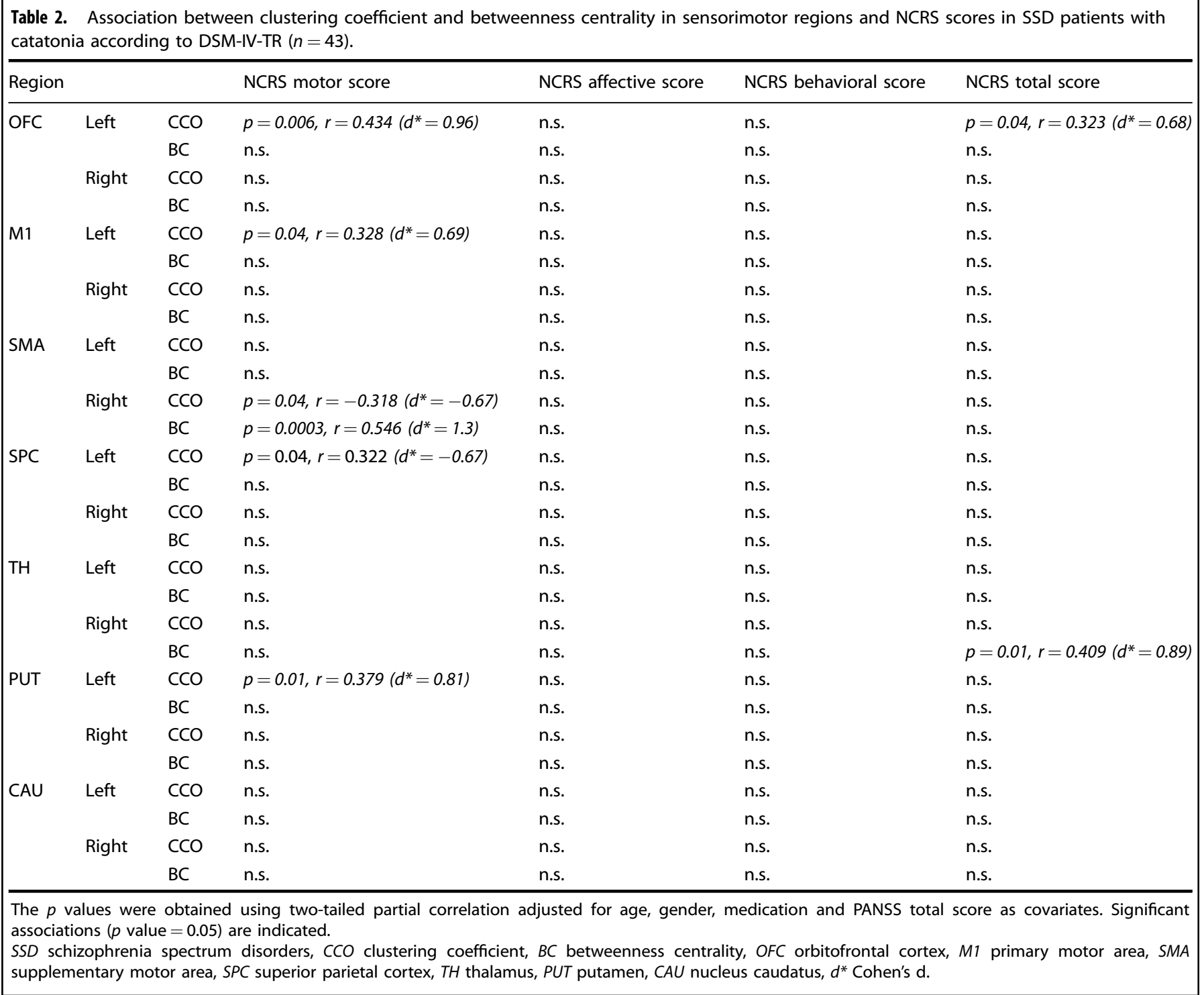

and colleagues showed that a very subtle hypomyelination in the prefrontal CC of $\mathrm{Mbp}^{+/-}$(heterozygous) mice (MBP myelin basic protein) is associated with age-related catatonia-like behavior [68]. Muetzel and colleagues found a significant association between WM microstructure in CC and bimanual task performance [69]. Second, CST contains monosynaptic connections (originating in M1) to spinal motoneurons, which are responsible for fine, fractionated movements of the hand [70-73]. Our findings suggest that reduced myelination of CC and CST might lead to less efficient neural signal transmission causing difficulties in movement execution and coordination $[69,74]$, as we can observe them in catatonia. Third, the T_PREM cortical fiber tract is part of the cerebellar-thalamo-cortical system, which is the major bundle through which the cerebellum affects voluntary movements in higher mammals [75, 76]. Interestingly, CST and T_PREM showed higher FA in catatonic compared to non-catatonic patients. These findings are in line with a very recent MRI study that used TBSS and examined WM abnormalities in schizophrenia patients with catatonia [77]. Viher and colleagues [77] identified 13 patients with ( $\geq 2$ items on the BFCRSI for a minimum of $24 \mathrm{~h}$ ) and 35 patients without catatonia. Patients with catatonia revealed leftlateralized higher FA in the CST and internal capsule when compared to non-catatonic patients. A possible explanation for the FA increase in CST may be that this structural reorganization is necessary to compensate for the impaired top-down modulation of higher-order cortical areas and aberrant motor behavior in catatonia $[77,78]$. Although striatal involvement in catatonia has only rarely been described in case reports $[23,24]$, it has been not detected by MRI studies focusing on GMV or resting-state activity. Therefore, it is rather a disturbed regional connectivity in the striatum that can lead to the development of catatonic symptoms. The finding of a significant association between FA variations in the left CST and NCRS behavioral score supports the abovementioned arguments and shows a specific relationship to behavioral abnormalities in the context of catatonic syndrome.

\section{Large-scale network analysis}

Using graph analytics, we identified significantly increased CCO of the right SCP and the left caudate nucleus in catatonic compared to non-catatonic patients. These findings could reflect regionally increased structural connectivity of SCP and caudate nucleus, which may account for catatonic motor symptom expression [12, 13]. However, these regions did not survive Bonferroni correction and should be considered as exploratory. Another important finding is the significant correlation between NCRS motor score and BC of the right SMA. This association did survive the correction for multiple 
testing and is noteworthy for several reasons: First, the SMA is a key region that subserves high-order internally initiated movements [79]. Second, this finding confirms prior studies that showed aberrant neural activity in the SMA of catatonic patients [60,80]. Third, these results are particularly consistent with the study by Walther and colleagues who found that excited catatonia was associated with higher perfusion of SMA [12]. Therefore, both aberrant activity as well as BC variations of the SMA may contribute to catatonic symptoms in SSD [9]. Taken together, these data suggest a key role of the SMA in the circuitry that has been previously implicated in catatonia, and suggest that one potential mechanism may be through deviations in the main top-down flow of information through cortical and subcortical sensorimotor circuits.

\section{Strengths and limitations}

The study sample size, well-matched study groups, and the use of comprehensive set of sophisticated WM microstructural parameters are the strengths of our study. However, some methodological aspects limit the generalizability of our results: First, the antipsychotic medication should be considered as a potential confounder. Second, no statement can be made regarding waxing and waning course of catatonia. The question concerning the symptom stability vs. dynamics could be robustly answered using longitudinal monitoring, preferably using electronic devices. Third, our DTI sequences didn't include the brainstem and cerebellum (in favor of higher resolution of cortical structures), so we cannot appreciate contributions of WM microstructural alterations in the brainstem and cerebellar tracts to catatonia [12]. Finally, we only examined SSD patients with catatonic symptoms. Since catatonia occurs in $9-17 \%$ of acute mental disorders [81] and is also present in specific medical conditions, we strongly advocate transdiagnostic MRI studies on catatonic symptoms. This approach will support the intensive effort to recognize catatonia as an independent diagnostic entity in future classification systems.

\section{CONCLUSION}

The present study provides consistent evidence for WM microstructure abnormalities of bundles connecting orbitofrontal/ parietal, thalamic and striatal regions in catatonia. The data suggest that catatonia is associated with widespread corticalsubcortical changes in structural connectivity that go beyond the previously reported atrophy of frontoparietal regions.

\section{FUNDING AND DISCLOSURE}

This work was supported by the German Research Foundation (DFG) (grant number DFG HI 1928/2-1 to DH, WO 1883/6-1 to RCW, MA 6340/10-1 and MA 6340/12-1 to KHM-H) and German Federal Ministry of Education and Research (BMBF, grant 01GQ1102 to HT). The DFG and BMBF had no further role in study design; in the collection, analysis and interpretation of data; in the writing of the report; and in the decision to submit the paper for publication. GN is grateful for financial support from PSI and CIHR in Canada. The authors have declared that there are no conflicts of interest in relation to the subject of this study.

\section{ACKNOWLEDGEMENTS}

We are grateful to all the participants and their families for their time and interest in this study.

\section{AUTHOR CONTRIBUTIONS}

DH, RCW and KMK: Design of the study. JW, KHM-H, PFN: Data analysis. DH, SF, AH, LSG and HT: Data collection. DH, RCW, JW, GN, KMK: Interpretation of the results, writing and manuscript revision.
Publisher's note Springer Nature remains neutral with regard to jurisdictional claims in published maps and institutional affiliations.

\section{REFERENCES}

1. Northoff G, Koch A, Wenke J, Eckert J, Boker H, Pflug B, et al. Catatonia as a psychomotor syndrome: a rating scale and extrapyramidal motor symptoms. Mov Disord: Off J Mov Disord Soc. 1999;14:404-16.

2. Fink M. Rediscovering catatonia: the biography of a treatable syndrome. Acta Psychiatr Scand Suppl. 2013;441:1-47.

3. Fink M, Shorter E, Taylor MA. Catatonia is not schizophrenia: Kraepelin's error and the need to recognize catatonia as an independent syndrome in medical nomenclature. Schizophr Bull. 2010;36:314-20.

4. Francis A, Fink M, Appiani F, Bertelsen A, Bolwig TG, Braunig P, et al. Catatonia in diagnostic and statistical manual of mental disorders, fifth edition. J Ect. 2010;26:246-7.

5. Wilson JE, Niu K, Nicolson SE, Levine SZ, Heckers S. The diagnostic criteria and structure of catatonia. Schizophrenia Res. 2015;164:256-62.

6. Kirby $\mathrm{GH}$. The catatonic syndrome and its relation to manic-depressive insanity. J Nerv Ment Dis. 1913;40:694-704.

7. Lange J. Katatonische Erscheinungen im Rahmen manisch-depressiver Erkrankungen. Berlin: Springer; 1922.

8. Hirjak D, Wolf RC, Northoff G GABA and negative affect-catatonia as model of RDoC-based investigation in psychiatry. Schizophr Bull. 2019;45:1168-69.

9. Hirjak D, Kubera KM, Wolf RC, Northoff G. Going back to Kahlbaum's psychomotor (and GABAergic) origins: is catatonia more than just a motor and dopaminergic syndrome? Schizophr Bull. 2020;46:272-85.

10. Hirjak D, Rashidi M, Kubera KM, Northoff G, Fritze S, Schmitgen MM, et al. Multimodal magnetic resonance imaging data fusion reveals distinct patterns of abnormal brain structure and function in catatonia. Schizophr Bull. 2020;46:202-10.

11. Hirjak D, Kubera KM, Northoff G, Fritze S, Bertolino AL, Topor CE, et al. Cortical contributions to distinct symptom dimensions of catatonia. Schizophr Bull. 2019;45:1184-94.

12. Walther S, Schappi L, Federspiel A, Bohlhalter S, Wiest R, Strik W, et al. Restingstate hyperperfusion of the supplementary motor area in Catatonia. Schizophr Bull. 2017;43:972-81.

13. Walther S, Stegmayer K, Federspiel A, Bohlhalter S, Wiest R, Viher PV. Aberrant hyperconnectivity in the motor system at rest is linked to motor abnormalities in schizophrenia spectrum disorders. Schizophr Bull. 2017;43:982-92.

14. Walther S, Stegmayer K, Wilson JE, Heckers S. Structure and neural mechanisms of catatonia. Lancet Psychiatry. 2019;6:610-19.

15. Ellul P, Choucha W. Neurobiological approach of catatonia and treatment perspectives. Front psychiatry. 2015;6:182.

16. Rogers JP, Pollak TA, Blackman G, David AS. Catatonia and the immune system: a review. Lancet Psychiatry. 2019;6:620-30.

17. Janova $H$, Arinrad $S$, Balmuth $E$, Mitjans $M$, Hertel J, Habes $M$, et al. Microglia ablation alleviates myelin-associated catatonic signs in mice. J Clin Invest. 2018;128:734-45.

18. Hagemeyer N, Goebbels S, Papiol S, Kastner A, Hofer S, Begemann M, et al. A myelin gene causative of a catatonia-depression syndrome upon aging. EMBO Mol Med. 2012;4:528-39.

19. Smith SM, Jenkinson M, Johansen-Berg H, Rueckert D, Nichols TE, Mackay CE, et al. Tract-based spatial statistics: voxelwise analysis of multi-subject diffusion data. Neurolmage. 2006;31:1487-505.

20. Wassermann D, Rathi $Y$, Bouix $S$, Kubicki $M$, Kikinis $R$, Shenton $M$, et al. White matter bundle registration and population analysis based on Gaussian processes. Inf Process Med Imaging. 2011;22:320-32.

21. Yeatman JD, Dougherty RF, Rykhlevskaia E, Sherbondy AJ, Deutsch GK, Wandell $B A$, et al. Anatomical properties of the arcuate fasciculus predict phonological and reading skills in children. J Cogn Neurosci. 2011;23:3304-17.

22. Wasserthal J, Neher PF, Hirjak D, Maier-Hein KH. Combined tract segmentation and orientation mapping for bundle-specific tractography. Med Image Anal. 2019;58:101559.

23. Tsujino N, Nemoto $T$, Yamaguchi $T$, Katagiri N, Tohgi N, Ikeda $R$, et al. Cerebral blood flow changes in very-late-onset schizophrenia-like psychosis with catatonia before and after successful treatment. Psychiatry Clin Neurosci. 2011;65:600-3.

24. Lauer M, Schirrmeister $H$, Gerhard A, Ellitok E, Beckmann $H$, Reske $S N$, et al. Disturbed neural circuits in a subtype of chronic catatonic schizophrenia demonstrated by F-18-FDG-PET and F-18-DOPA-PET. J Neural Transm (Vienna). 2001;108:661-70.

25. Goch CJ, Stieltjes B, Henze R, Hering J, Poustka L, Meinzer HP, et al. Quantification of changes in language-related brain areas in autism spectrum disorders using large-scale network analysis. Int J Comp Assisted Radiol Surg. 2014;9:357-65. 
26. Freeman LC. A set of measures of centrality based on betweenness. Sociometry. 1977;40:31-40.

27. Hirjak D, Thomann PA, Northoff G, Kubera KM, Wolf RC NCR-Skala - Deutsche Version der Northoff Catatonia Rating Scale (NCRS-dv) - Ein validiertes Messinstrument zur Erfassung katatoner Symptome. Der Nervenarzt. 2016:(im Druck).

28. Ungvari GS, Caroff SN, Gerevich J. The catatonia conundrum: evidence of psychomotor phenomena as a symptom dimension in psychotic disorders. Schizophrenia Bull. 2010;36:231-8.

29. Heckers S, Tandon R, Bustillo J. Catatonia in the DSM-shall we move or not? Schizophr Bull. 2010;36:205-7.

30. Oldfield RC. The assessment and analysis of handedness: the Edinburgh inventory. Neuropsychologia. 1971;9:97-113.

31. Sass H, Wittchen HU, Zaudig MIH. Diagnostisches und Statistisches Manual Psychischer Störungen DSM-IV-TR: Textrevision. Hogrefe Verlag; Auflage: 1 (1. Januar 2003); 2003.

32. Leucht S, Samara M, Heres S, Patel MX, Furukawa T, Cipriani A, et al. Dose equivalents for second-generation antipsychotic drugs: the classical mean dose method. Schizophr Bull. 2015;41:1397-402.

33. Dazzan P, Morgan KD, Orr KG, Hutchinson G, Chitnis X, Suckling J, et al. The structural brain correlates of neurological soft signs in AESOP first-episode psychoses study. Brain. 2004;127:143-53.

34. Gay O, Plaze M, Oppenheim C, Mouchet-Mages S, Gaillard R, Olie JP, et al. Cortex morphology in first-episode psychosis patients with neurological soft signs. Schizophr Bull. 2013;39:820-9.

35. Jenkinson M, Beckmann CF, Behrens TE, Woolrich MW, Smith SM. Fsl. Neuroimage. 2012;62:782-90.

36. Woolrich MW, Jbabdi S, Patenaude B, Chappell M, Makni S, Behrens T, et al. Bayesian analysis of neuroimaging data in FSL. Neuroimage. 2009;45:S173-86.

37. Veraart J, Novikov DS, Christiaens D, Ades-Aron B, Sijbers J, Fieremans E. Denoising of diffusion MRI using random matrix theory. Neuroimage. 2016;142:394-406.

38. Kellner E, Dhital B, Kiselev VG, Reisert M. Gibbs-ringing artifact removal based on local subvoxel-shifts. Magn Reson Med: Off J Soc Magn Reson Med / Soc Magn Reson Med. 2016;76:1574-81.

39. Andersson JLR, Sotiropoulos SN. An integrated approach to correction for offresonance effects and subject movement in diffusion MR imaging. Neuroimage. 2016;125:1063-78.

40. Tustison NJ, Avants BB, Cook PA, Zheng Y, Egan A, Yushkevich PA, et al. N4ITK: improved N3 bias correction. IEEE Trans Med Imaging. 2010;29:1310-20.

41. Smith SM. Fast robust automated brain extraction. Hum brain Mapp. 2002;17:143-55.

42. Jenkinson $M$, Smith S. A global optimisation method for robust affine registration of brain images. Med Image Anal. 2001;5:143-56.

43. Bach M, Laun FB, Leemans A, Tax CMW, Biessels GJ, Stieltjes B, et al. Methodological considerations on tract-based spatial statistics (TBSS). Neurolmage. 2014;100:358-69.

44. Smith SM, Nichols TE. Threshold-free cluster enhancement: addressing problems of smoothing, threshold dependence and localisation in cluster inference. Neurolmage. 2009;44:83-98.

45. Kelly S, Jahanshad N, Zalesky A, Kochunov P, Agartz I, Alloza C, et al. Widespread white matter microstructural differences in schizophrenia across 4322 individuals: results from the ENIGMA Schizophrenia DTI Working Group. Mol Psychiatry. 2018;23:1261-69.

46. Wasserthal J, Neher P, Maier-Hein KH. TractSeg - Fast and accurate white matter tract segmentation. Neuroimage. 2018;183:239-53.

47. Nichols TE, Holmes AP. Nonparametric permutation tests for functional neuroimaging: a primer with examples. Hum brain Mapp. 2002;15:1-25.

48. Yeatman JD, Dougherty RF, Myall NJ, Wandell BA, Feldman HM. Tract profiles of white matter properties: automating fiber-tract quantification. PLoS One. 2012;7: e49790.

49. Neher PF, Descoteaux M, Houde JC, Stieltjes B, Maier-Hein KH. Strengths and weaknesses of state of the art fiber tractography pipelines-A comprehensive invivo and phantom evaluation study using Tractometer. Med Image Anal. 2015;26:287-305.

50. Rubinov M, Bullmore E. Fledgling pathoconnectomics of psychiatric disorders. Trends Cogn Sci. 2013;17:641-7.

51. Fischl B, Dale AM. Measuring the thickness of the human cerebral cortex from magnetic resonance images. Proc Natl Acad Sci USA. 2000;97:11050-5.

52. Fischl B, Sereno MI, Dale AM. Cortical surface-based analysis. II: inflation, flattening, and a surface-based coordinate system. Neurolmage. 1999;9:195-207.

53. Dale AM, Fischl B, Sereno MI. Cortical surface-based analysis. I. Segmentation and surface reconstruction. Neurolmage. 1999;9:179-94.

54. Fischl B, van der Kouwe A, Destrieux C, Halgren E, Segonne F, Salat DH, et al. Automatically parcellating the human cerebral cortex. Cereb Cortex. 2004;14:11-22.
55. Desikan RS, Segonne F, Fischl B, Quinn BT, Dickerson BC, Blacker D, et al. An automated labeling system for subdividing the human cerebral cortex on MRI scans into gyral based regions of interest. Neurolmage. 2006;31:968-80.

56. Segonne F, Dale AM, Busa E, Glessner M, Salat D, Hahn HK, et al. A hybrid approach to the skull stripping problem in MRI. Neurolmage. 2004;22:1060-75.

57. Smith RE, Tournier JD, Calamante F, Connelly A. The effects of SIFT on the reproducibility and biological accuracy of the structural connectome. Neuroimage. 2015;104:253-65.

58. Barnes J, Ridgway GR, Bartlett J, Henley SM, Lehmann M, Hobbs N, et al. Head size, age and gender adjustment in MRI studies: a necessary nuisance? Neuroimage. 2010;53:1244-55.

59. Hyatt CS, Owens MM, Crowe ML, Carter NT, Lynam DR, Miller JD. The quandary of covarying: a brief review and empirical examination of covariate use in structural neuroimaging studies on psychological variables. Neuroimage. 2020;205:116225.

60. Payoux P, Boulanouar K, Sarramon C, Fabre N, Descombes S, Galitsky M, et al Cortical motor activation in akinetic schizophrenic patients: a pilot functional MRI study. Mov Disord: Off J Mov Disord Soc. 2004;19:83-90.

61. Mamah D, Ji A, Rutlin J, Shimony JS. White matter integrity in schizophrenia and bipolar disorder: Tract- and voxel-based analyses of diffusion data from the Connectom scanner. Neuroimage Clin. 2019;21:101649.

62. Huttlova J, Kikinis Z, Kerkovsky M, Bouix S, Vu MA, Makris N, et al. Abnormalities in myelination of the superior cerebellar peduncle in patients with schizophrenia and deficits in movement sequencing. Cerebellum. 2014;13:415-24.

63. Gulani V, Webb AG, Duncan ID, Lauterbur PC. Apparent diffusion tensor measurements in myelin-deficient rat spinal cords. Magn Reson Med. 2001:45:191-5.

64. Kubicki M, McCarley RW, Shenton ME. Evidence for white matter abnormalities in schizophrenia. Curr Opin psychiatry. 2005;18:121-34.

65. Kubicki M, Westin CF, McCarley RW, Shenton ME. The application of DTI to investigate white matter abnormalities in schizophrenia. Ann N. Y Acad Sci. 2005;1064:134-48.

66. Kubicki M, Park H, Westin CF, Nestor PG, Mulkern RV, Maier SE, et al. DTI and MTR abnormalities in schizophrenia: analysis of white matter integrity. Neuroimage. 2005;26:1109-18.

67. D'Agati E, Casarelli L, Pitzianti MB, Pasini A. Overflow movements and white matter abnormalities in ADHD. Prog Neuro-Psychopharmacol Biol psychiatry. 2010;34:441-5.

68. Poggi G, Boretius S, Mobius W, Moschny N, Baudewig J, Ruhwedel T, et al. Cortical network dysfunction caused by a subtle defect of myelination. Glia. 2016;64:2025-40.

69. Muetzel RL, Collins PF, Mueller BA, Lim AMS, Luciana KO. M. The development of corpus callosum microstructure and associations with bimanual task performance in healthy adolescents. Neurolmage. 2008;39:1918-25.

70. Porter R. Lemon R. Corticospinal Function and Voluntary Movement. Oxford, NY: Clarendon Press, Oxford University Press; 1993.

71. Witham $\mathrm{CL}$, Fisher KM, Edgley SA, Baker SN. Corticospinal Inputs to Primate Motoneurons Innervating the Forelimb from Two Divisions of Primary Motor Cortex and Area 3a. J Neurosci: Off J Soc Neurosci. 2016;36:2605-16.

72. Bopp MHA, Zollner R, Jansen A, Dietsche B, Krug A, Kircher TJ. White matter integrity and symptom dimensions of schizophrenia: a diffusion tensor imaging study. Schizophr Res. 2017;184:59-68.

73. Wu L, Calhoun VD, Jung RE, Caprihan A. Connectivity-based whole brain dual parcellation by group ICA reveals tract structures and decreased connectivity in schizophrenia. Hum brain Mapp. 2015;36:4681-701.

74. Forssberg $\mathrm{H}$, Eliasson AC, Kinoshita $\mathrm{H}$, Johansson RS, Westling $\mathrm{G}$. Development of human precision grip. I: Basic coordination of force. Exp brain Res. 1991;85:451-7.

75. Nashef A, Cohen O, Israel Z, Harel R, Prut Y. Cerebellar shaping of motor cortical firing is correlated with timing of motor actions. Cell Rep. 2018;23:1275-85.

76. Nashef A, Rapp H, Nawrot MP, Prut Y. Area-specific processing of cerebellarthalamo-cortical information in primates. Biol Cybern 2018;112:141-52.

77. Viher PV, Stegmayer K, Federspiel A, Bohlhalter S, Wiest R, Walther S. Altered diffusion in motor white matter tracts in psychosis patients with catatonia. Schizophr Res. 2020;12:S0920-9964:30120-1. https://doi.org/10.1016/j.schres.2020.03.017. epub ahead of print.

78. Northoff G. What catatonia can tell us about "top-down modulation": a neu ropsychiatric hypothesis. Behav Brain Sci. 2002;25:555-77.

79. Lara AH, Cunningham JP, Churchland MM. Different population dynamics in the supplementary motor area and motor cortex during reaching. Nat Commun. 2018;9:2754.

80. Scheuerecker J, Ufer S, Kapernick M, Wiesmann M, Bruckmann H, Kraft E, et al. Cerebral network deficits in post-acute catatonic schizophrenic patients measured by fMRI. J Psychiatr Res. 2009;43:607-14.

81. Rasmussen SA, Mazurek MF, Rosebush PI. Catatonia: Our current understanding of its diagnosis, treatment and pathophysiology. World J Psychiatry. 2016;6:391-98. 